\title{
Assistência farmacêutica em relação ao uso off-label de medicamentos no âmbito da pandemia do COVID-19
}

\author{
Pharmaceutical assistance in relation to off-label use of medicines under the COVID-19 pandemic
}

Asistencia farmacéutica en relación con el uso fuera de la etiqueta de medicamentos en el marco de

la pandemia COVID-19

Recebido: 12/11/2021 | Revisado: 20/11/2021 | Aceito: 27/11/2021 | Publicado: 09/12/2021

\author{
Érika Pinheiro Silva \\ ORCID: https://orcid.org/0000-0002-3061-8966 \\ Faculdade de Palmas, Brasil \\ E-mail: erikapinheiro238@gmail.com \\ Maykon Jhuly Martins de Paiva \\ ORCID: https://orcid.org/0000-0002-6228-4550 \\ Faculdade de Palmas, Brasil \\ E-mail: maykonjhuly@hotmail.com
}

\begin{abstract}
Resumo
O objetivo deste trabalho é abordar os desafios e a importância da assistência farmacêutica na disseminação de informação, bem como analisar a influência da mídia sobre o aumento do consumo de medicamentos, durante a pandemia da COVID-19. O presente estudo trata-se de uma revisão bibliográfica, onde foram realizadas pesquisas através das bases de dados SCIELO (Scientific Eletronic Library Online), LILACS (Literatura Latino Americana de Ciência da Saúde), Google Acadêmico, Organização Mundial da Saúde, Organização Pan-Americana de Saúde, e Conselho Federal de Farmácia. Em 11 de março de 2020, a OMS declarou formalmente a existência de uma pandemia causada por um novo coronavírus, o SARS-CoV-2; devido ao momento épico e assustador surgiram diversas crenças e informações infundadas em medicamentos milagrosos que preveniria e até curaria a COVID-19, em alguns estados foram distribuídos os chamados "kit COVID-19". Este tema está no contexto da saúde pública, em escala mundial, especialmente, no que se refere ao uso racional de medicamentos, envolvendo a segurança consumidores e as prescrições off-label. O aumento no consumo de medicamentos off-label durante a pandemia é notório. A OMS e Anvisa precisam se atentar que efeitos potenciais negativos de um medicamento sem comprovação cientifica para determinada enfermidade, podem trazer sérios riscos futuros.
\end{abstract}

Palavras-chave: Pandemia; COVID-19; Medicamentos Off-label; Uso racional; Assistência farmacêutica.

\begin{abstract}
The objective of this paper is to address the challenges and the importance of pharmaceutical assistance in disseminating information, as well as analyzing the influence of the media on the increase in drug consumption during the COVID-19 pandemic. The present study is a literature review, where searches were carried out through the databases SCIELO (Scientific Electronic Library Online), LILACS (Latin American Health Science Literature), Academic Google, World Health Organization, Pan- American Health, and Federal Council of Pharmacy. On March 11, 2020, WHO formally declared the existence of a pandemic caused by a new coronavirus, SARS-CoV-2; due to the epic and frightening moment, several beliefs and unfounded information emerged in miraculous medicines that would prevent and even cure COVID-19, in some states the so-called "COVID-19 kit" were distributed. This theme is in the context of public health, on a global scale, especially with regard to the rational use of medicines, involving consumer safety and off-label prescriptions. The increase in off-label drug consumption during the pandemic is notorious. WHO and Anvisa need to be aware that potential negative effects of a drug without scientific evidence for a given disease, can bring serious future risks.
\end{abstract}

Keywords: Pandemic; COVID-19; Off-label drugs; Rational use; Pharmaceutical care.

\section{Resumen}

El objetivo de este trabajo es abordar los desafíos y la importancia de la asistencia farmacéutica en la difusión de información, así como analizar la influência de los medios de comunicación en el aumento del consumo de drogas durante la pandemia de COVID-19. El presente estudio es una revisión de la literatura, donde se realizaron búsquedas a través de las bases de datos SCIELO (Scientific Electronic Library Online), LILACS (Latin American Health Science Literature), Academic Google, Organización Mundial de la Salud, Pan-American Health y Federal Council of Pharmacy. El 11 de marzo de 2020, la OMS declaró formalmente la existencia de una pandemia causada por un nuevo coronavirus, el SARS-CoV-2; Debido al épico y espantoso momento, surgieron várias creencias e información 
infundada en medicinas milagrosas que prevenirían e incluso curarían el COVID-19, en algunos estados se distribuyó el llamado "kit COVID-19". Este tema está en el contexto de la salud pública, a escala mundial, especialmente en lo que respecta al uso racional de los medicamentos, que involucra la seguridad del consumidor y las prescripciones no autorizadas. El aumento en el consumo de medicamentos no aprobados durante la pandemia es notorio. La OMS y Anvisa deben ser conscientes de que los posibles efectos negativos de un medicamento sin evidencia científica para una enfermedad determinada pueden traer graves riesgos en el futuro.

Palabras clave: Pandemia; COVID-19; Medicamentos no aprobados; Uso racional; Atención farmacéutica.

\section{Introdução}

Em dezembro do ano 2019, no hospital de Wuhan, na China, surgem os primeiros casos de COVID-19 no mundo, como uma pneumonia de causa desconhecida. Dois meses depois, mais especificamente, no dia 09 de fevereiro de 2020 , trinta e quatro brasileiros que viviam na cidade chinesa Wuhan, ponto central do novo coronavírus, foram repatriados. Duas aeronaves da Força Aérea Brasileira aterrissaram no Brasil com o grupo, e os mesmos, ficaram de quarentena por 14 dias na Base aérea de Anápolis em Goiás (Croda \& Garcia; Lana et al, 2020).

Enquanto isso, o Ministério da Saúde ampliava a lista de países em alerta, e monitorava casos suspeitos de infecção pelo novo coronavírus. Até que, no dia 26 de fevereiro de 2020, é confirmado o primeiro caso de contaminação pelo vírus denominado SARS-CoV-2, no Brasil; o paciente se trata de um homem de 61 anos que viajou à Itália, e deu entrada no Hospital Albert Einstein, em São Paulo - SP, no dia anterior. Daí em diante os casos foram aumentando cada vez mais e o País entrou em estado de alerta, era o início de tempos difíceis e desafiadores para o sistema governamental e de saúde, assim como para a população em geral, que teriam que aprender a conviver e lidar com algo desconhecido e de alta contaminação (Vellano \& Paiva 2020).

Com a disseminação do causador da COVID-19 pelo mundo, em 11 de março de 2020, o diretor-geral da OMS declarou pandemia, e negociou com o Poder Legislativo a liberação de recurso para ações de enfrentamento ao coronavírus, oriundos de emendas, com o intuito de envolver governantes e a sociedade em ações de prevenção da infeção, minimizar os efeitos e salvar vidas (OMS, 2020).

Desde início do surto mundial do Sars-Cov-2 se fez necessário medidas de urgência para o tratamento, controle, e promoção a saúde; para atender às demandas do momento pandêmico, cientistas de todo o mundo se mobilizaram na busca por novos tratamentos, e o reposicionamento de fármacos surgiu naturalmente como a alternativa mais viável, sucedendo a uma gama de propostas de prevenção e terapias medicamentosas, alguns estudos baseados na opinião de especialistas, alguns em resultados in vitro promissores, e outros em descobertas em séries de casos de tratamentos compassivos ou não recomendados (Lana et al, 2020).

Funestamente, ao serem submetidos aos rigorosos processos de ensaios clínicos randomizados, poucas evidências de benefícios tangíveis permanecem; devido à falta de um medicamento comprovadamente eficaz que trate e cure a infecção pelo SARS-CoV- 2, as medidas de combate à COVID-19 se constituem da realização de quarentena, vacinação e buscas por drogas capazes de reverterem a forma grave da doença (Ferreira \& Andricopulo, 2020; Chen et al, 2021; Holanda, 2020).

A pandemia da COVID-19 surgiu de forma repentina apresentando diversos desafios, não só no Brasil, mas no mundo todo, um momento épico que será lembrado em toda a história da humanidade como um mal que levou milhares de vidas e assolou os viventes do século XXI, sendo este um momento nunca vivenciado desde a constituição do de Saúde (SUS).

Profissionais de Saúde sofrem com as incertezas e com a sua vulnerabilidade, assim, o presente trabalho se propôs a analisar e descrever o enfrentamento e a história do Vírus SARS-CoV-2, bem como as dificuldades enfrentadas pelos profissionais, em especial o profissional farmacêutico, e, o uso indiscriminado de medicamentos, de forma off-label, em busca de alternativas, com a intenção de contribuir para uma melhor compreensão sobre barreiras e facilitadores pertinentes ao tema (Sanar Saúde, 2012). 


\section{Metodologia}

O presente estudo constitui em uma pesquisa bibliográfica narrativa, que, segundo Clandinin e Connely (2000, p. 20) pode ser definida como "uma forma de entender a experiência" em um processo de colaboração entre pesquisador e pesquisado. A pesquisa narrativa mais comum pode ser descrita como uma metodologia que consiste na coleta de histórias sobre determinado tema onde o investigador encontrará informações para entender determinado fenômeno tem como objetivo analisar a realidade a partir da observação da pandemia. Onde foram coletados dados através das bases SCIELO (Scientific Eletronic Library Online), LILACS (Literatura Latino-Americana de Ciência da Saúde), Google Acadêmico, Google, através da consulta de artigos científicos e matérias publicadas pela Organização Mundial da Saúde, Organização Pan-Americana de Saúde, Conselho Federal de Farmácia, e livros.

Figura 1: Resultados da pesquisa realizada nas bases de dados.

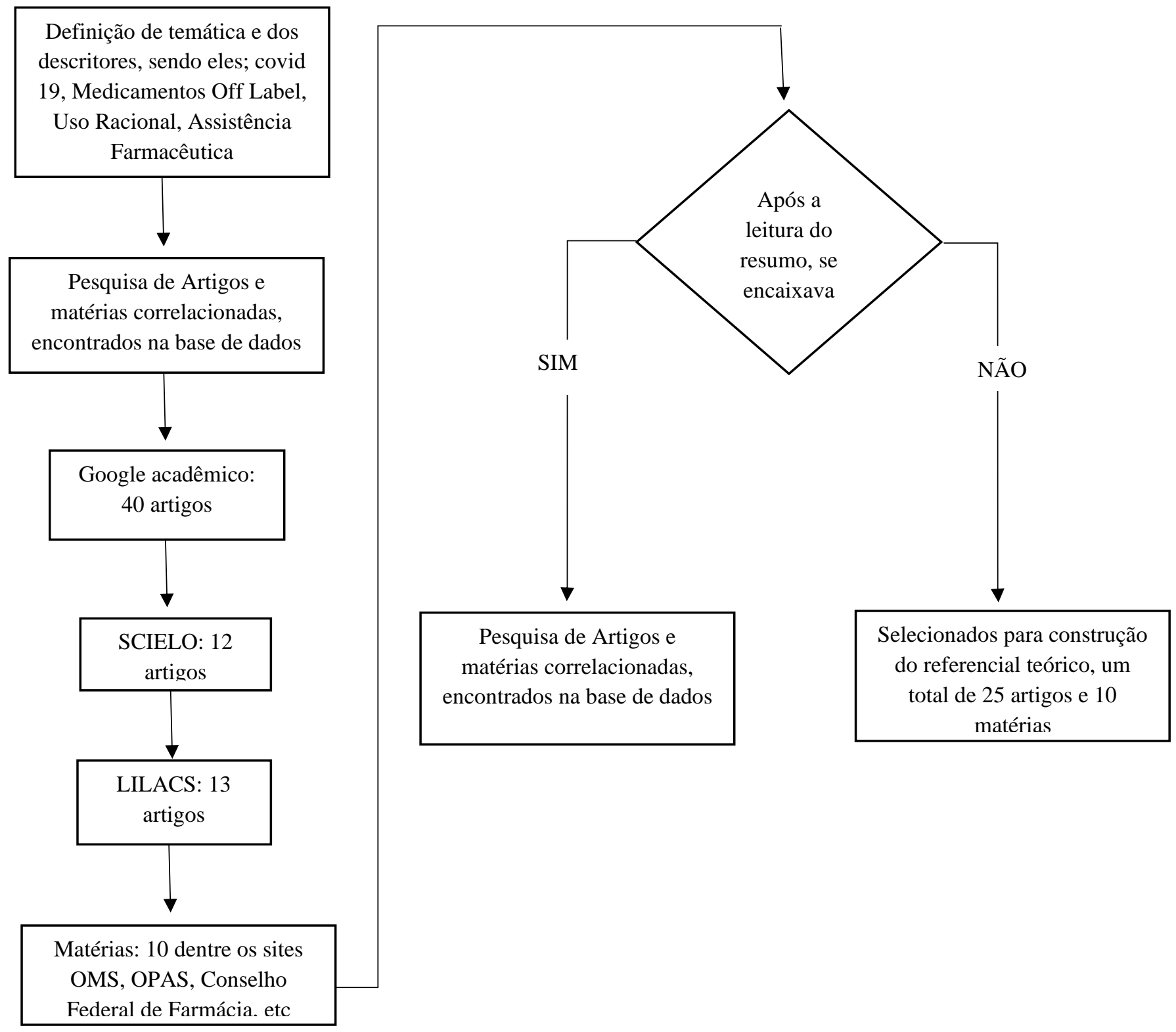

Fonte: Autores (2021). 
Para o levantamento bibliográfico foram utilizados dados relacionados ao tema, independente do ano, com os seguintes descritores: Pandemia, Medicamentos Off-label, Uso Racional e Assistência farmacêutica; sobre a COVID-19, a pesquisa foi limitada entre os anos de 2019 à 2021; sendo a mesma realizada de fevereiro a setembro deste mesmo ano, onde foram selecionados 40 artigos e 10 matérias relacionadas, que estavam de acordo com os critérios de inclusão da temática em questão.

Como critério de inclusão foram selecionados artigos originais, nos idiomas português, espanhol e inglês, pertinentes ao tema, como critério de exclusão foram utilizados os artigos duplicados, trabalhos sem metodologias e fontes claras, e que não se enquadraram nos descritores buscados.

Quadro 1. Referências distribuídas pelos eixos temáticos.

\begin{tabular}{|c|c|}
\hline Eixos Temáticos & Referências \\
\hline $\begin{array}{l}\text { COVID-19 e } \\
\text { Impactos da } \\
\text { Pandemia }\end{array}$ & $\begin{array}{l}\text { Angonesi, D. \& Sevalho, G. 2010; Aquino, E. M. L. et al. 2020; Chen, X., Liao, B., Cheng, L., Peng, X., Xu, } \\
\text { X., Li, Y., ... \& Ren, B. (2020); Croda, J. H. R., \& Garcia, L. P. (2020); de Jesus Pereira, A, Narduchi, F., \& } \\
\text { de Miranda, M. G. (2020); Holanda, V. N. 2020; Lana, R. M., Coelho, F. C., Gomes, M. F. D. C., Cruz, O. } \\
\text { G., Bastos, L. S., Villela, D. A. M., \& Codeço, C. T. (2020); Lopes, M. L. D. D. S., \& Lima, K. C. D. 2021; } \\
\text { Lopez-Leon, S., Wegman-Ostrosky, T., Perelman, C., Sepulveda, R., Rebolledo, PA, Cuapio, A., \& } \\
\text { Villapol, S. 2021; Mariani, F., \& Mattos, M. 2012; Sanar Saúde, } 2012\end{array}$ \\
\hline $\begin{array}{l}\text { Atenção e Serviços } \\
\text { Farmacêuticos }\end{array}$ & $\begin{array}{l}\text { Conselho Nacional de Saúde (Brasil), Resolução n 338, 2004; CFF, Brasilia, p. 1-44, 2015; Cordeiro, H. A } \\
\text { indústria da saúde no Brasil. Rio de Janeiro: Graal; 1980; Dos Passos, M. M. B., de Moraes Castoldi, V., \& } \\
\text { Soler, O. 2021; Pinheiro, R. M. 2010; Rdc n 44/2009 Lei Federal n 13.021/2014; Santos, M. R. D. 1993; } \\
\text { Saturnino, L. T. M., Perini, E., Luz, Z. M. P. D., \& Modena, C. M. } 2012\end{array}$ \\
\hline $\begin{array}{l}\text { Uso Off-label e } \\
\text { Racional de } \\
\text { medicamentos }\end{array}$ & $\begin{array}{l}\text { Aquino, D. S. D. 2008; Carvalho, M. L. 2016; Claverie, H. 2016; Da Silveira, M. C. 2019; Ferreira, L. L., \& } \\
\text { Andricopulo, A. D. 2020; Ismp-Institudo Para Práticas Seguras No Uso De Medicamentos. 2018; Leandro, J. } \\
\text { A., \& Santos, F. L. 2015; Ministerio Da Saúde (Brasil). 2012; Paumgartten, F. J. R., \& Oliveira, A. C. A. X. } \\
\text { D. 2020; Silva, A. F. M. (2018); Silva, J.P. da, Batista, L. de O 2020; Silva, M.E.H. \& Abreu, C. R. C. 2021; } \\
\text { Vellano, P. O \& Paiva, M. J. M. de. } 2020\end{array}$ \\
\hline
\end{tabular}

Fonte: Autores (2021).

\section{Resultados e Discussão}

\section{O uso off-label de medicamentos durante a pandemia da COVID-19 e possíveis impactos.}

O uso de medicamentos off-label está relacionado a utilização para indicações diferentes daquelas que consistem no resumo das características do medicamento (RCM), seja na dosagem, subgrupo populacional, forma de administração ou indicação terapêutica definida pela entidade reguladora, que ao lançar um medicamento no mercado, desenvolve todo um conhecimento acerca daquele fármaco, baseado em experimentos sobre seus efeitos e sua toxicidade em animais (estudos préclínicos) e, caso não se observem efeitos tóxicos intoleráveis, fazem-se os primeiros ensaios clínicos em humanos, que são denominadamente conhecidos como estudos de fase I, II e III, para investigar possíveis aspectos relacionados com a toxicidade, farmacocinética e eficácia em seres humanos (Carvalho, 2016; Silva A, 2018).

Uma das consequências mais trágicas da prescrição off-label e acontecimento decisivo na criação de um sistema regulador de medicamentos fora desenvolvida comercialmente pela companhia farmacêutica Chemie Grunenthal, da Alemanha Ocidental, tendo grande sucesso como sonífero e antiemético, propriedades observadas nos testes realizados pela referida companhia, com base nas alegações de segurança do fabricante, o produto foi anunciado pela empresa como 'atóxico', era consumido e vendido livremente, sem prescrição médica, como um tranquilizante para melhorar o sono e expandido para gestantes, pois melhorava o enjoo matinal, além de diversas outras finalidades, tais como irritabilidade, baixa concentração, estado de pânico, ejaculação precoce, tensão pré- menstrual, medo de ser examinado, depressão etc. (Claverie, 2016; Leandro \& Santos, 2015).

Logo, após o consumo exacerbado começou a surgir os danos, dentre os principais, a malformação congênita dos bebês, 
deixando um marco na história devido a essa tragédia em massa; nessa época, os ensaios clínicos nos EUA não exigiam a aprovação ou supervisão da U.S. Food and Drug Administration (FDA), este é o incidente que levou à passagem da Emenda sobre drogas de Kefauver-Harris; já no final da década de 1990, começaram a surgir prescrições incluindo uma combinação de dois medicamentos utilizados para a perda de peso, fenfluramina e fentermina, combinação esta intitulada de "Fen-Phen", que aparentava ser uma receita de perda de peso milagrosa.

Entretanto, no ano 1997, a FDA afastou os medicamentos do mercado, depois de descobrir que esta combinação causava problemas a nível das válvulas cardíacas. Assim, é possível deduzir, tomando-se o exemplo da talidomida e da fenfluramina e fentermina, que efeitos potenciais negativos de um medicamento sem comprovação cientifica para determinada enfermidade, podem trazer riscos. Mesmo depois de todos esses grandes acontecimentos e já existindo um rigoroso processo de aprovação de medicamentos criado, há momentos em que a prescrição off- label continua a acarretar graves preocupações com a saúde pública, como este que estamos vivendo, onde se há prescrições de diversos medicamentos off-label sem saber as consequências e causas futuras (Claverie, 2016; Leandro \& Santos, 2015).

Devido ao momento e as incertezas, surgiram diversas crenças e informações infundadas em medicamentos milagrosos que preveniria e até curaria a COVID-19. Na procura por soluções rápidas, em alguns estados foram distribuídos os chamados "kit COVID- 19" contendo azitromicina, ivermectina e cloroquina ou hidroxicloroquina (Lopes \& Lima, 2021).

Outros candidatos como os antivirais ritonavir e lopinavir, o antiparasitário nitazoxanida e o corticoide dexametasona também foram propagados, dentre tantos outros que são temas de discussão em todo o mundo; A procura por resultados simples parece não ter limites, são indicadas diversas classes terapêuticas, dentre as principais investigadas estão: antivirais, anticâncer, anti-hipertensivos, imunossupressores, antiparasitários, anti-inflamatórios, fitoterápicos, entre outras que mesmo sem comprovação científica confirmada ainda tem sido amplamente utilizadas para a prevenção ou tratamento de pessoas com sintomas iniciais da doença. Lidar com uma infecção na qual não existe terapia imediata e eficaz traz dúvidas não apenas para leigos, mas também para médicos e profissionais de saúde, que acabam adotando medidas que não se baseiam nas melhores informações cientificas (Paumgartten \& Oliveira, 2020).

O impacto ocasionado pela pandemia do coronavírus impôs grandes mudanças na rotina diária da população brasileira, não somente na área da saúde, mas diversos outros ramos foram diretamente atingidos, como a educação, economia, entre tantos outros; todos os setores começaram a definir critérios de prevenção e contágio do vírus, passando a ter que se reinventar e criar medidas para continuar as atividades rotineiras.

Na educação, uma das medidas tomadas foi a inclusão da EAD nas escolas de ensino básico e superior, como alternativa à interrupção das atividades presenciais; na economia, devido ao isolamento social necessário, empresas tiveram que adotar o modelo de trabalho home-office, no qual funcionários mantiveram o vínculo empregatício formal com a empresa, porém, trabalhando de forma remota, onde os avanços tecnológicos da comunicação e tecnologia contribuíram consideravelmente, e apesar da flexibilização na forma de trabalho foi possível seguir uma rotina; além de tantas outras empresas que não tiveram como manter seus seguimentos, acarretando na redução no quadro de funcionários e consequentemente, demissões. Esta crise causada pela COVID-19, que o mundo enfrentou e ainda vem superando, fez a população entrar em estado emocional grave, acarretando o consumo demasiado e errôneo de medicamentos, tanto os industrializados quanto os remédios naturais (de Jesus Pereira et al., 2020; Silva J \& Batista, 2020).

A pratica do uso off-label traz uma série de dilemas, ainda mais em um momento pandêmico onde se tem um vírus de alta transmissão que faz milhares de vítimas todos os dias e ainda não há de fato uma cura para a doença em questão; de um lado, a prática médica deve levar em consideração todas as alternativas terapêuticas, o que justifica a prescrição off- label em situações onde não há escolhas, de outro lado, os riscos de intoxicações, resistências bacterianas, agravamento do caso clinico do paciente, entre outros fatores de risco que também necessitam ser levados em consideração (da Silveira, 2019; Applied Microbiology And 
Biotechnology et al., 2020).

\section{Uso racional de medicamentos}

A Organização Mundial de Saúde (OMS) estabelece o uso racional de medicamentos através de trajetórias que deverão ser seguidas, sendo elas: estabelecer a necessidade do uso do medicamento apropriados para cada condição clínicas, em forma farmacêutica e doses adequadas às suas necessidades particulares, por um período adequado, que esteja disponível de modo oportuno com menor custo para quem usa e para a comunidade, com a necessária orientação e responsabilidade na prescrição e dispensação, a fim de que se cumpra o regime terapêutico e obtenha resultados (OMS, 2020).

Em contraposição a promoção da OMS, quando um ou mais desses pressupostos não são atendidos, caracteriza se o uso irracional de medicamentos, um problema recorrente a nível mundial. Estima-se que mais de $50 \%$ de todos os medicamentos são prescritos ou dispensados de forma impropria e que metade de todos os pacientes não os utiliza adequadamente, alguns exemplos desse uso inadequado são: uso desnecessário de muitos medicamentos pelo chamado "paciente polifarmácia" (aqueles que fazem o uso rotineiro de quatro ou mais medicamentos, com ou sem prescrição médica); uso indevido de antibiótico, muitas vezes para infecções não bacterianas e em dosagens erradas; uso demasiado de medicamentos injetáveis quando por via oral seria mais propicia e segura, não adesão e frequência do tratamento, além da automedicação (Ministério Da Saúde, 2012; Ismp, 2018).

No livro A indústria da saúde no Brasil, de 1980, Cordeiro afirma que medicamentos ocupam lugares simbólicos e representações que obscurecem os determinantes sociais das doenças, iludem os indivíduos com a aparência de eficácia científica e, como mercadoria, realizam o valor e garantem a acumulação de um dos seguimentos mais lucrativos do capital industrial" baseado nesta fala, Destaca-se o aumento na quantidade de prescrições de antibióticos, anti-inflamatórios e outras classes terapêuticas já relatadas neste trabalho, especialmente em pacientes hospitalizados, no intuito de prevenir/tratar infecções secundárias à COVID-19 (Barros, 2004).

A busca por respostas fáceis e imediata ao sofrimento, faz com que a população em geral considere esses medicamentos uma "luz no fím do túnel" se sentindo atraídos. Porém, a crença excessiva, e de certa forma ingênua no poder dos medicamentos, ao lado da crescente oferta e indicação desses produtos pela mídia e indústria farmacêutica, tende a aproximar ainda mais a sociedade ao consumo, de forma irracional, como se fosse uma solução mágica para o mal que aflige a atualidade (Barros, 2004).

A intenção de propor e considerar-se todas as alternativas em um momento de caos e alerta de saúde pública, incialmente, parece ser boa, porém, tem seu preço, que não se restringe ao desembolso financeiro, mas a homeostasia e saúde do corpo, em meio a promessas de prevenção e cura midiáticas duvidosas, que no decorrer dos estudos não se tem comprovações cientificas, devemos considerar que existe uma ecologia do corpo, que merece ser preservada e poupada da poluição e intervenções farmacológicas desnecessárias, pois o mesmo medicamento que cura, pode deixar danos irreversíveis ou até mesmo matar (Aquino D. \& Aquino E et al. , 2008)

\section{Papel do farmacêutico no enfretamento da pandemia COVID-19 e disseminação de informações corretas Breve histórico}

A origem do termo fármaco vem de farmácia, que é designado do grego pharmakon, e tinha duplo significado: medicamento e veneno. Como diz o físico Paracelsus (1493 - 1541), “todas as substâncias são veneno, não há uma que não seja. A posologia correta diferencia o veneno do remédio."

Desde a antiguidade, a Farmácia sempre se fez presente na vida do homem, principalmente nos piores momentos, aquele da busca do alívio da dor, aflição e cura. No princípio, os medicamentos eram produzidos de forma artesanal nas boticas e pequenas indústrias e baseava-se apenas em experiencias práticas dos profissionais que atuavam sem diplomação, como os curandeiros, raizeiros e benzedores que prometiam a cura e tinham a confiança da população; isso, até o ano de 1832, quando se 
implantou a primeira escola de farmácia no Brasil (Santos, 1993).

As chamadas boticas, onde farmacêutico trabalhava com preparados magistrais principalmente de origem vegetal ou animal, indicava e orientava quanto ao uso correto e exerciam grande importância econômica e social nas cidades brasileiras, devido ao alto potencial comercial; Com a industrialização do medicamento e grandes descobertas terapêuticas como as dos antimicrobianos, impulsionaram a comunidade científica, consequentemente proporcionando um crescimento do setor industrial, nascendo desta forma, o medicamento moderno, racional e cientificamente explicado, a produção artesanal foi desaparecendo e dando lugar a produção em larga escala, feita pelas indústrias; tendo os profissionais farmacêuticos que se reinventar e mudar o seu foco, que até então, era exclusivo do paciente e da produção de medicamentos (Santos, 1993; Angonesi e Sevalho, 2010)

\section{Desafios da Assistência Farmacêutica}

Em consonância com as novas tendências do mercado de trabalho e a reformulação da profissão, as diretrizes curriculares, o farmacêutico está apto a desempenhar suas funções com qualidade, nas 131 especialidades distribuídas em 10 áreas de atuação regulamentadas pela Resolução/CFF no 572/2013; exercendo atividades referentes aos fármacos e aos medicamentos, às análises clínicas e toxicológicas e ao controle, produção e análise de alimentos, bem como atuação em princípios éticos e na compreensão da realidade social, cultural e econômica do seu meio, dirigindo sua atuação para a transformação da realidade, em benefício da sociedade, fazendo parte de uma equipe multidisciplinar que envolve a formação e a prática dos profissionais da saúde em favor do bem-estar e da qualidade de vida das pessoas (Conselho Federal De Farmácia, 2015; Saturnino et al., 2012)

No Brasil, a AF (assistência farmacêutica) e o que se a construção das iniciativas para efetivação dessa assistência como área de atuação do sistema de saúde, só foi criada após 10 anos do estabelecimento do SUS, nesse espaço de tempo gerou-se lacunas e repressões culminando em diversas dificuldades e desafios que se refletem ainda nos dias de hoje, pois, apesar dos farmacêuticos serem os profissionais capacitados e responsáveis na área dos medicamentos, ainda se limitam ao controle e distribuição, e aos que ousam ser diferentes e ir além desse papel, são muitas vezes desacreditados. (Vieira, 2008.)

Juntamente com inovação tecnológica, a sociedade também mudou seus costumes; os produtos que eram feitos de forma artesanal e vendidos em pequenas quantidades, a indústria modificou e incrementou com propagandas que incentivam o consumo, e esse, no atual momento, é um dos principais desafios da AF no âmbito da COVID-19, pois a prática de prescrever medicamentos registrados para uso que não estão incluídos nas informações do produto (off label), juntamente com a propagação pelos canais de mídia, fazem com que a população, em busca de alternativas, aumente a procura desses medicamentos, e consequentemente, o uso não racional dos mesmos (Silva \& Abreu, 2021; Saturnino et al., 2012).

Segundo a RDC n 44/2009 Lei Federal n 13.021/2014, Ministério da Saúde, dentre as boas práticas farmacêuticas estão: conjunto de técnicas e medidas de segurança, manutenção da qualidade dos produtos e serviços ofertados em farmácias e drogarias, assistência e atenção farmacêutica com informação aos cidadãos, e promoção a saúde, contribuindo com o uso racional de medicamentos e melhora na qualidade de vida dos usuários, conduzindo de forma ética e segura.

Na atual situação de Saúde Pública, o farmacêutico deve estar preparado para enfrentamento da doença e repasse de informações e orientações corretas, visto que a farmácia comunitária, na maioria das vezes é o primeiro lugar onde os pacientes buscam um tratamento ao apresentarem sintomas de doenças comuns, tais como: dor de cabeça, dor de garganta, febre, congestionamento nasal, espirros, tosse e náuseas, entre outros. Nesses casos, é muito frequente a prática da automedicação, um hábito que traz diversos riscos à saúde, e cabe ao profissional alertar quanto ao uso racional e aos perigos dessa prática tão comum. Deve também, prestar Serviços Farmacêuticos, difundindo informações científicas em linguagem acessível, voltados à promoção do Uso Racional de Medicamentos (URM), através de estratégias de educação em saúde, que envolve uma prescrição adequada, o acesso oportuno e a utilização, com intervalo e tempo definidos, de medicamentos custo-efetivos, seguros, eficazes 
e de qualidade, visando à prevenção de riscos maiores, à promoção, proteção e recuperação da saúde dos pacientes (RDC n ${ }^{\circ}$ 44/2009; dos Passos et al., 2021)

No ambiente hospitalar, se faz a junção da assistência técnica-clínica e administrativas onde se realizam atividades relacionadas à assistência farmacêutica, à produção, ao armazenamento, ao controle, à dispensação, à distribuição de medicamentos e correlatos às unidades hospitalares, assim como a assistência a paciente, sempre em busca da adesão e eficácia terapêutica (dos Passo et al., 2021)

\section{Cuidados farmacêuticos no pós COVID}

A síndrome respiratória causada pelo SARS-CoV-2, pode perdurar e envolver sequelas e outras complicações médicas que duram semanas a meses após a recuperação inicial. Embora a comunidade cientifica não estarem medindo esforços em busca de alternativas e respostas, ainda é cedo para afirmar os efeitos a longo prazo, e existe a possibilidade de efeitos que ainda não foram detectados, porém, o que se nota até os dias atuais é que a maioria dos sintomas pós-COVID se assemelha à sintomatologia desenvolvida durante a fase aguda da doença, podendo gerar sequelas de natureza pneumologia, neurológica, dermatológica, psicológica, etc; sendo os efeitos mais comuns: fadiga, redução da capacidade pulmonar, dor de cabeça, anosmia, problemas de memória, distúrbio de atenção, queda de cabelo e dispneia. (Lopez-Leon et al.,2021)

De acordo com a Resolução ํo 338 de 04 de maio de 2004, e como já relatado anteriormente neste trabalho, a assistência farmacêutica trata-se de um conglomerado de ações voltadas à promoção proteção e recuperação da saúde dos indivíduos de maneira singular, tanto quanto coletivamente, tendo o medicamento como insumo fundamental e visando o acesso e ao seu uso consciente. Conjunto este que envolve a pesquisa, o desenvolvimento e a produção de medicamentos e insumos, bem como a sua seleção, programação, aquisição, distribuição, dispensação, garantia da qualidade, acompanhamento na perspectiva da obtenção de resultados, avaliação e melhoria da qualidade de vida da população. Devidamente implementado na Rede de atenção primaria a saúde junto ao Sistema Único de Saúde (SUS), bem como nas farmácias comunitárias, com enfoque na melhoria de vida do paciente e estabelecimento de uma relação terapêutica, os impactos positivos e melhor serão os resultados na adesão ao tratamento.

O farmacêutico participa de um processo de assistência ao paciente onde tem a importante responsabilidade de suprir as necessidades e sanar as dúvidas em relação aos medicamentos, podendo adotar diversas metodologias para melhor compreensão do paciente e prevenção de outros danos; no que se refere ao pós-COVID (Silva \& Araújo, 2020).

O profissional pode atuar no acompanhamento neuropsicológico em relação a farmacoterapia adotada pelo prescritor, bem como busca de alternativas não farmacológicas para atribuir e melhorar a saúde mental pacientes com transtornos psicológicos; avaliação da capacidade respiratória dos pacientes através de testes oximetria de pulso, medição da pressão arterial e do pico do fluxo expiratório por Peak Flow , bem como outras ações educativas, de orientações e informação para recuperação da homeostasia do paciente. Nesse sentido, os riscos associados à terapêutica e agravamento dos sintomas podem ser minimizados, evitando transtornos mais graves para o paciente e para o sistema de saúde (Pinheiro, 2010).

\section{Conclusão}

O presente estudo retratou alguns fatos históricos sobre o uso Off-label e irracional de medicamentos, produto este que, além de ajudar no processo de cura de vários tipos de enfermidades, exercem importante papel econômico para os fabricantes, distribuidores, comerciantes e para o País, como um todo; porém, como consequência, em um cenário pandêmico, como este, a falta de informação e o uso incorreto pode incidir em grandes complicações, contribuindo com o aumento da morbidade e mortalidade atribuídas a infecção, o que causa profundas preocupações no setor da saúde, pois, apesar de existirem evidências suficientes de estudos observacionais e clínicos que mostraram que os efeitos adversos graves superam notoriamente os 
benefícios clínicos, ainda são propagadas informações infundidas e sem comprovações cientificas. Dentro deste cenário, o farmacêutico poderá contribuir com a diminuição das consequências do uso incorreto de medicamentos através da sua atuação na definição dos cuidados com os indivíduos, orientando no ato da dispensação quanto ao uso racional de medicamentos e no controle deles, além de contribuir com a equipe multidisciplinar na identificação das consequências fisiológicas e psicológicas pós-COVID-19. Por se tratar de uma doença nova e com descobertas diárias, observa-se várias limitações nas experiências narradas nos estudos sobre temática, bem como neste; dentre elas a inexperiência dos profissionais da saúde que lidam diretamente com a doença, a alta mutação do vírus, poucos estudos concretos publicados na área, a vagarosidade na implementação de ações eficazes, entre outros.

\section{Referências}

Angonesi, D. \& Sevalho, G. (2010). Atenção Farmacêutica: fundamentação conceitual e crítica para um modelo brasileiro. Ciênc. saúde coletiva 15(suppl 3), 112. https://doi.org/10.1590/S1413-81232010000900035.

https://www.scielosp.org/article/ssm/content/raw/?resource_ssm_path=/media/assets/csc/ v15s3/v15s3a35.pdf.

Aquino, D. S. D. (2008). Por que o uso racional de medicamentos deve ser uma prioridade? Ciência \& Saúde Coletiva, 13, 733-736. http://www.cienciaes audecoletiva.com.br/artigos/por-que-o-uso-racional-de-medicamentos-deve-ser-uma-prioridade/1509?id=1509

Aquino, E. M. L. et al. (2020). Ciência \& Saúde Coletiva. Medidas de distanciamento social no controle da pandemia de COVID-19: potenciais impactos e desafios no Brasil. https://www.scielosp.org/article/csc/2020.v25supp11/2423- 2446/pt/

Barros, J. A. C. (2004). Políticas farmacêuticas: a serviço dos interesses da saúde? Brasília: UNESCO/ANVISA. https://www.cff.org.br/userfiles/file/n oticias/Politicas\%20Farmaceuticas\%20Versao\%20Final.pdf

Carvalho, M. L. (2016). O desafio do uso off label de medicamentos. Revista Paulista de Pediatria. 34(1), 1-2 http://dx.doi.org/10.1016/j.rppede.2015.12.007.

Chen, X., Liao, B., Cheng, L., Peng, X., Xu, X., Li, Y., \& Ren, B. (2020). A coinfecção microbiana em COVID-19. Microbiologia aplicada e biotecnologia, 19. https://link.springer.com/content/pdf/10.1007/s00253-020-10814-6.pdf.

Claverie, H. (2016). On-Label vs. Off-Label Drug Prescribing. IG Living, p. 1-4, 10. http://www.igliving.com/magazine/articles/IGL_2016-12_AR_On-Labelvs-Off-Label-Prescribing.pdf.

Conselho Nacional de Saúde (Brasil), Resolução no 338, de 6 de maio de 2004. https://bvsms.saude.gov.br/bvs/saudelegis/cns/2004/res0338_06_05_2004.html

Conselho Federal De Farmácia (Brasil). Perfil do Farmacêutico no Brasil. CFF, Brasilia, 1-44, 2015. https://www.cff.org.br/userfiles/file/Perfil\%20 do\%20farmac\%C3\%AAutico\%20no\%20Brasil\%20_web.pdf.

Cordeiro, H. A indústria da saúde no Brasil. Graal; 1980.

Croda, J. H. R., \& Garcia, L. P. (2020). Resposta imediata da Vigilância em Saúde à epidemia da COVID-19. Epidemiologia e Serviços de Saúde, 29(1). https://doi.org/10.5123/S1679-49742020000100021.

Da Silveira, M. C. (2019). O uso Off Label de Medicamentos no Brasil. Dissertação em Saúde Pública) - Escola Nacional de Saúde Pública Sergio Arouca, Brasília. https://www.arca.fiocruz.br/handle/icict/39683

de Jesus Pereira, A, Narduchi, F., \& de Miranda, M. G. (2020). Biopolítica e Educação: os impactos da pandemia do COVID-19 nas escolas públicas. Revista Augustus, 25(51), 219-236.https://revistas.unisuam.edu.br/index.php/revistaaugustus/article/view/554/299.

Dos Passos, M. M. B., de Moraes Castoldi, V., \& Soler, O. (2021). O papel do farmacêutico na pandemia de COVID-19: Revisão integrativa. Research, Society and Development, 10(6), e27110615809-e27110615809. https://rsdjournal.org/index.php/rsd/article/view/15809/14085.

Ferreira, L. L., \& Andricopulo, A. D. (2020). Medicamentos e tratamentos para a COVID-19. Estudos Avançados, 34, 7-27 https://doi.org/10.1590/s01034014.2020.34100.002.

Holanda, V. N. (2020). Pandemia de COVID-19 e os esforços da ciência para combater o novo coronavírus. Revista Interfaces: Saúde, Humanas e Tecnologia, 8(1), 360-361. http://interfaces.leaosampaio.edu.br/index.php/revista-interfaces/article/view/748

Ismp-Institudo Para Práticas Seguras No Uso De Medicamentos. (2018). Orgulho em Promover a Segurança do Paciente. Desprescrição - reduzindo a polifarmácia e prevenindo erros de medicação. https://www.ismp-brasil.org/site/noticia/desprescricao-reduzindo-a-polifarmacia-e-prevenindo-erros-demedicacao/

Lana, R. M., Coelho, F. C., Gomes, M. F. D. C., Cruz, O. G., Bastos, L. S., Villela, D. A. M., \& Codeço, C. T. (2020). Emergência do novo coronavírus (SARSCoV-2) e o papel de uma vigilância nacional em saúde oportuna e efetiva. Cadernos de Saúde Pública,36, e00019620. https://doi.org/10.1590/0102$311 \mathrm{X} 00019620$.

Leandro, J. A., \& Santos, F. L. (2015). História da talidomida no Brasil a partir da mídia impressa (1959-1962) 1. Saúde e Sociedade, 24, 991-1005. https://www.scielosp.org/pdf/sausoc/2015.v24n3/991-1005/pt. 
Research, Society and Development, v. 10, n. 16, e128101623246, 2021

(CC BY 4.0) | ISSN 2525-3409 | DOI: http://dx.doi.org/10.33448/rsd-v10i16.23246

Lopes, M. L. D. D. S., \& Lima, K. C. D. (2021). A pandemia COVID-19 e os erros na condução da sua abordagem em termos populacionais. https://www.scielo.br/j/rbgg/a/MZ3ZXgWrQbpBdTZNfbK7LPB/?lang=pt.

Lopez-Leon, S., Wegman-Ostrosky, T., Perelman, C., Sepulveda, R., Rebolledo, P. A., Cuapio, A., \& Villapol, S. (2021). Mais de 50 efeitos de longo prazo do COVID-19: uma revisão sistemática e meta-análise. Relatórios científicos, 11 (1), 16144. https://doi.org/10.1038/s41598-021-95565-8

Mariani, F., \& Mattos, M. (2012). Clandinin, D. Jean; Connelly, F. Michael. Pesquisa narrativa: experiência e história em pesquisa qualitativa. Tradução: Grupo de Pesquisa Narrativa e Educação de Professores ILEEI/UFU. Uberlândia: EDUFU, 2011. 250 p. Revista De Educação Pública, 21(47), 663-667. Recuperado de https://periodicoscientificos.ufmt.br/ojs/index.php/educacaopublica/article/view/1766

Ministerio Da Saúde (Brasil). (2012). Uso racional de medicamentos. Bvsms.saude, Brasilia, (1), 1-158, https://bvsms.saude.gov.br/bvs/public acoes/uso_racional_medicamentos_temas_selecionados.pdf.

Paiva, V. L. M. D. O. (2008). A pesquisa narrativa: uma introdução. Revista brasileira de linguística aplicada, 8, 261-266. https://doi.org/10.1590/S198463982008000200001

Paumgartten, F. J. R., \& Oliveira, A. C. A. X. D. (2020). Uso off label, compassivo e irracional de medicamentos na pandemia de COVID-19, consequências para a saúde e questões éticas. Ciência \& Saúde Coletiva, 25(9), 3413-3419. https://www.scielo.br/j/csc/a/GQwLcBQZmGYp7mK3V6r7tFt/?la ng=en\&format=pdf

Pinheiro, R. M. (2010). Serviços farmacêuticos na atenção primária à saúde. Tempus Actas de Saúde Coletiva, 4(3), 15. https://tempusactas.unb.br/inde x.php/temp us/article/view/874/837.

Rdc nº 44/2009 Lei Federal n 13.021/2014, Ministério da Saúde. https://www.mypharma.com.br/blog/rdc-44-09/

Sanar Saúde. (2012). Sanar med. Linha do tempo do Coronavírus no Brasil. [S.l.]. Disponível em: https://www.sanarmed.com/linha-do-tempo-do-coronavirusno-brasil.

Santos, M. R. D. (1993). Do boticário ao bioquímico: as transformações ocorridas com a profissão farmacêutica no Brasil. https://pesquisa.bvsalud.org/p ortal/resource/pt/lil-117231

Saturnino, L. T. M., Perini, E., Luz, Z. M. P. D., \& Modena, C. M. (2012). Farmacêutico: um profissional em busca de sua identidade. https://www.arca.fiocruz.br/bitstream/icict/7860/1/Farmac\%c3\%aautico.pdf.

Silva, A. F. M. (2018). Uso off-label de medicamentos: um tema controverso. Dissertação de Mestrado. Universidade do Alvarge, Portugal. http://hdl.handle.net/10400.1/12508

Silva, J. P. da, \& Batista, L. de O. de. Impactos da automedicação em tempos de pandemia COVID-19. In: Tomasi, C. D., Soratto, J., Ceretta, L. B. (org.). Interfaces da COVID-19: impressões multifacetadas do período de pandemia. Criciúma, SC: UNESC, 2020. 74-75. http://repositorio.unesc.net/handle/1/7823.

Silva, M. E. H. \& Abreu, C. R. C. (2021). Medicamentos off label. Revista JRG De Estudos Acadêmicos, 4(8), 300-309. https://doi.org/10.5281/zenodo.4641121https://doi.org/10.5281/zenodo.4641121

Vellano, P. O., \& Paiva, M. J. M. de. (2020). O uso de antimicrobiano na COVID-19 e as infecções: o que sabemos. Research, Society and Development, 9(9), e841997245. https://doi.org/10.33448/rsd-v9i9.7245 